\title{
Méta-analyse et efficacité des psychothérapies :
}

\author{
Faits et fictions
}

\section{Meta-analysis and psychotherapy efficacy :}

\section{Facts and fictions}

Grégoire Zimmermann \& Valentino Pomini

LabDCI, Institut de Psychologie, Université de Lausanne, SUISSE

Correspondance : Prof. Grégoire Zimmermann, $\mathrm{PhD}$

Institut de Psychologie, Faculté des Sciences Sociales et Politiques

Université de Lausanne

Quartier Mouline - Bâtiment Geopolis

CH-1015 Lausanne, SUISSE

E-mail: Gregoire.Zimmermann@unil.ch

Tel. : +41 (0)2169232 60, Fax : +41(0)216923265 


\title{
Méta-analyse et efficacité des psychothérapies : Faits et fictions
}

\section{Résumé}

Dans un contexte de plus en plus marqué par des impératifs d'ordre économique, la question de l'évaluation de l'efficacité des psychothérapies fait aujourd'hui encore débat. Historiquement, nous trouvons les origines de cette question au début des années 50 lorsque Eysenck (1952), dans un article désormais célèbre, affirmait que la psychothérapie était dénuée d'efficacité. Devant l'abondance des résultats, Glass (1976) a développé la méthode de la méta-analyse, qui a pour objectif de combiner les résultats de plusieurs essais cliniques contrôlées afin d'en faire une synthèse reproductible et quantifiée. Suite à une brève présentation historique, cet article a pour objectif de définir les principes de la méta-analyse, de la contextualiser épistémologiquement, et enfin de discuter de l'intérêt et des limites de l'application de cette méthode dans le champ de l'évaluation des psychothérapies.

Mots-clefs : psychothérapie, efficacité, méta-analyse, méthodologie, traitements psychologiques appuyés empiriquement

\section{Meta-analysis and psychotherapy efficacy : Facts and fictions}

\begin{abstract}
In a political environment increasingly concerned with the problem of escalating health care costs, the issue of psychotherapy efficacy evaluation is still debated. Historically, this debate started after Eysenck (1952) published a famous article suggesting that average improvement from pre- to post-therapy has nothing to do with psychotherapy participation, but something that would tend to happen anyway ("spontaneous remission"). Wanting to prove that psychotherapy was effective (Glass, 2000), Smith and Glass (1977) published the first meta-analysis of the psychotherapy efficacy, combining the results of several controlled clinical trials, and found that psychotherapy was remarkably efficacious. Following a brief
\end{abstract}


historical introduction, the objectives of this paper is to define the principles of meta-analysis, to discuss of epistemological contextualization of this methodological approach, and finally to examine the interest and limits of the application of this method in the field of evaluation of psychotherapy.

Keywords : psychotherapy, efficacy, meta-analysis, methodology, evidence-based psychology 


\section{Introduction}

Dans un contexte marqué par des impératifs économiques et politiques de réduction des coûts de la santé, la question de l'efficacité des psychothérapies fait aujourd'hui toujours débat. Pendant longtemps, l'évaluation empirique de l'effet des psychothérapies a pourtant semblé peu en rapport avec les enjeux cliniques de ce type de prise en charge : nombre d'écoles thérapeutiques estimaient qu'il était réducteur sinon impossible de procéder à une évaluation quantitative de l'effet des psychothérapies. Toutefois, le succès important au cours des vingt dernières années des mouvements de l'Evidence-Based Medecine (EBM) et de l'Evidence-Based Practice (EBP) en psychologie ont activement contribué à un changement de perspective (pour l'EBM, voir Sacket, Rosenberg, Gray, Haynes, et Richardson, 1996 ; pour l'EBP, voir APA Task Force on Evidence-Based Practice, 2006).

Historiquement, l'origine de cette question remonte au début des années 50 lorsque Eysenck (1952) affirmait sur la base d'une revue de vingt-quatre études publiées que la psychothérapie (notamment psychanalytique) était dénuée d'efficacité. En effet, les données de son étude l'amenaient à conclure sans équivoque : les résultats à disposition « échouent à démontrer que la psychothérapie, freudienne ou autre, facilite le rétablissement des patients névrotiques. Ils indiquent qu'à peu près deux tiers des patients névrotiques récupèrent ou s'améliorent sensiblement dans les deux ans qui suivent l'apparition de leur trouble, qu'ils soient suivis ou pas en psychothérapie. (...) Du point de vue du névrosé, ces chiffres sont encourageants ; du point de vue du psychothérapeute, ils ne peuvent guère être invoqués à l'appui de leurs revendications ${ }^{1} »($ Eysenck, 1952, pp. 322-323). Suite à cette publication, nombre d'auteurs réagirent (e.g. Luborsky, 1954 ; Rosenzweig, 1954) et des dizaines d'études alimentèrent la controverse (par exemple Bergin, 1971 qui proposa une nouvelle analyse des données utilisées par Eysenck), sans pour autant clore définitivement le débat (pour un bref historique en français de ces controverses, voir Villamaux, 2000). Eysenck (1961, 1965,

\footnotetext{
${ }^{1}$ Traduction de l'auteur
} 
1983) lui-même, sans doute stimulé par le succès de son article original, publia plusieurs revues dans lesquels il tenta à nouveau de discréditer les psychothérapies psychodynamiques et « éclectiques » et d'établir l'efficacité de la thérapie comportementale.

Afin d'aborder ces questions d'une manière plus adéquate que ne l'avait fait Eysenck, Luborsky, Singer et Luborsky (1975) proposèrent la méthode du « box score » (box score method) qui consiste à déterminer dans un premier temps des critères d'inclusion des recherches à examiner (réduisant la subjectivité du chercheur dans le choix des études), et à comparer ensuite les résultats thérapeutiques des études sélectionnées en comptant le nombre de fois qu'une forme de thérapie est significativement supérieure, inférieure ou égale à une autre. A la même époque, devant l'accroissement général des résultats de recherche en sciences de l'éducation et en psychologie, Gene V. Glass (1976) développa la méthode de la méta-analyse (« l'analyse d'analyses »), qui a pour objectif de combiner les résultats de plusieurs études indépendantes et de permettre une synthèse quantifiée des résultats sur un problème donné. Une année plus tard, il publia avec sa collègue Mary Lee Smith une étude dans laquelle ils appliquèrent la méthode de la méta-analyse à la question de le l'efficacité des psychothérapies (Smith et Glass, 1977). Sur la base de 375 études comprenant environ 25000 participants, ils conclurent aux effets bénéfiques de la psychothérapie (Smith et Glass, 1977 ; Glass, 2000). Plus spécifiquement, leurs résultats indiquaient que $75 \%$ des patients non-traités ont des résultats inférieurs à la moyenne des patients bénéficiant d'une psychothérapie (Smith et Glass, 1977). Depuis, malgré les tentatives virulentes d'Eysenck (1978) de discréditer la méthode qu'il qualifiait d' " énorme bêtise » (mega-silliness), la méta-analyse a été très largement utilisée afin de faire la synthèses de résultats empiriques en médecine, psychologie ou sciences sociales.

Plus de quarante ans après, quels enseignements peut-on tirer de l'application de la métaanalyse dans le champ de l'évaluation des psychothérapies ? Qu'a-t-elle apporté ? Quelles 
sont ses limites et comment la situer dans ce large débat? L'objectif de cette contribution est de répondre brièvement à ces questions.

\section{Définition de la méta-analyse et application dans la recherche en psychothérapie}

La méta-analyse est une méthode quantitative qui permet de combiner les résultats de plusieurs études pour en faire une synthèse reproductible et quantifiée (Glass, 1976 ; Hunter et Schmidt, 1990). Cette démarche permet d'obtenir une vision globale des apports de la recherche empirique dans un domaine donné. L'indice statistique utilisé pour standardiser les résultats des différents travaux et les rendre comparable est la taille de l'effet (Effect size) (pour une présentation détaillée, voir par exemple Lipsey et Wilson, 2001). Les statistiques inférentielles habituellement utilisées indiquent si une différence observée peut être attribuée ou non au hasard. La taille de l'effet, quant à elle, permet de quantifier l'amplitude des différences observées, et contrairement aux tests statistiques classiques, elle est indépendante de la taille de l'échantillon. En général, selon les valeurs standards proposées par Cohen (1988), on considère qu'une taille de l'effet ( $d$ de Cohen - indice très fréquemment utilisé dans l'évaluation des psychothérapies) de 0,80 signale une différence importante entre le groupe contrôle et le groupe expérimental (lequel a bénéficié de la psychothérapie), de 0,50 une différence moyenne et de 0,20 une différence faible. Par exemple, si l'on s'intéresse à l'efficacité des psychothérapies pour le traitement du syndrome de stress post-traumatique, les résultats d'une méta-analyse récente mettent en évidence des tailles de l'effet de 1,11 et de 0,83 lorsque l'on compare respectivement la psychothérapie à une condition contrôle du type « liste d'attente » et à une condition contrôle du type traitement de soutien (Bradley, Greene, Russ, Dutra, et Westen, 2005). Exprimés différemment, ces $d$ de Cohen de 1,11 et de 0,83 indiquent respectivement qu'environ $86 \%$ des individus sur une liste d'attente et $79 \%$ des individus avec un traitement de soutien ont de moins bon résultats en moyenne que ceux qui bénéficient d'une prise en charge psychothérapeutique spécifique. Afin de rendre plus aisé 
l'interprétation de la taille de l'effet, Rosenthal et Rubin (1982) ont proposé un indice, le Binomial effect size display (BESD), qui permet d'exprimer une taille de l'effet en termes de différence de taux de succès entre les individus du groupe expérimental (p.ex. ceux ayant bénéficié de la psychothérapie) et ceux groupe contrôle (i.e. ceux qui n'ont pas eu de traitement). Ainsi un $d$ de 0,80 indique une augmentation du taux de réussite de la prise en charge d'environ 38\% entre le traitement contrôle et la psychothérapie (voir le tableau 1) (Randolph et Edmonson, 2005 ; Rosenthal, 1994).

- Insérer par ici Tableau I -

Même si les résultats d'une étude mettent en évidence une différence statistiquement significative, une taille de l'effet faible doit nuancer l'optimisme, indiquant au lecteur que, malgré la signification statistique, la différence s'avère au final plutôt réduite. L'appréciation de l'effet d'un traitement devrait ainsi toujours être discuté en fonction du type de traitement évalué et des bénéfices escomptés. Une taille de l'effet faible ne signifie donc pas nécessairement qu'un traitement n'est pas digne d'intérêt. Rosenthal $(1990,1994)$ rappelle à ce sujet l'exemple célèbre d'une essai clinique randomisé en double aveugle qui avait pour objectif d'examiner les effets de l'aspirine dans la prévention des maladies cardiovasculaires (p.ex. infarctus du myocarde). A première vue, les résultats de cette étude suggéraient que le bénéfice de l'aspirine en terme de taille de l'effet était trivial tant il était faible $(d=0,07)$. Cet effet correspond toutefois à une réduction de $3.5 \%$ du risque d'accidents cardiovasculaires qui n'est de loin pas négligeable en termes de santé publique. Du reste, cette étude fut prématurément interrompue dans la mesure où les auteurs jugèrent qu'il n'était plus éthiquement défendable que les médecins participant à cette étude (plus de 11000 médecins) continuent à prescrire un placebo à leurs patients (Steering Committee of the Physicians' Health Study Research Group, 1988). 
Dans le champ de la psychothérapie, les résultats des premières études méta-analytiques mettent en évidence des tailles de l'effet de l'ordre de 0.80 , alors que les travaux récents méthodologiquement plus rigoureux estiment que la taille de l'effet de la psychothérapie est plutôt de l'ordre de 0.65 (Lambert et Ogles, 2004 ; Grissom, 1996). Comparé à d'autres interventions thérapeutiques dans le domaine médical (p.ex. statines dans la prévention secondaire de l'infarctus du myocarde, voir Zimmermann, de Roten, et Despland, 2008), les résultats actuels indiquent que les psychothérapies représentent un type de traitement remarquablement efficace (efficacité absolue) pour une grande diversité de troubles psychiques (par exemple pour la dépression, les troubles anxieux ou encore les troubles de la personnalité) (pour une revue, voir Carr, 2009 ; Cooper, 2008 ; Roth et Fonagy, 2005).

\section{Intérêts et limites de la méta-analyse}

Il est aujourd'hui généralement admis que la méta-analyse est une des méthodologies les plus intéressantes en recherche quantitative ${ }^{2}$ afin d'effectuer la synthèse des données disponible sur une thématique donnée. Par rapport à une revue de littérature classique purement discursive, la méta-analyse se présente donc comme une méthodologie rigoureuse dont la démarche est transparente. Elle a notamment l'avantage d'être une démarche argumentée, reproductible, qui tient compte de la nature probabiliste des résultats d'études individuelles et qui permet une synthèse quantifiée de l'effet d'un traitement (Borenstein, Hedges, Higgins, et Rothstein, 2009 ; Cucherat, 1997)

Malgré l'intérêt de ce type de méthodologie dans l'évaluation des psychothérapies, un certain nombre de limites méritent néanmoins d'être évoquées. Premièrement, de manière générale, les méta-analyses s'inscrivent dans la mouvance initiée aux Etats-Unis par la division 12 (psychologie clinique) de l'American Psychological Association (APA) qui a développé des critères empiriques pour évaluer l'efficacité des traitements psychologiques et établir ce que l'on nomme les « traitements psychologiques appuyés empiriquement »

\footnotetext{
${ }^{2}$ A noter que l'équivalent existe en recherche qualitative (méta-étude qualitative ; Paterson, 2001)
} 
(empirically supported therapies ; EST) (Chambless et Hollon, 1998). Dans cette perspective, l'essai contrôlé randomisé (randomized controlled trial) et la méta-analyse constituent les dispositifs empiriques privilégiés pour examiner de façon rigoureuse l'efficacité des traitements psychologiques. Ces dispositifs quasi-expérimentaux, bien qu'utiles à la démarche évaluative, ne sont pas exempts de biais et présentent des limites importantes notamment lorsqu'il s'agit de considérer les questions de validité externe et d'applicabilité dans la pratique clinique quotidienne (question de l'efficience) (Nathan, Stuart, et Dolan, 2000 ; Rosqvist, Thomas, et Truax, 2011). A cet égard, un des éléments pertinents souvent occulté est la différence parfois très importante que l'on observe entre le nombre de cas examinés pour l'inclusion dans un essai contrôlé et le nombre effectif d'individus traités et évalués (biais de sélection). Ce constat nous amène à nous questionner sur la représentativité des individus inclus dans ces essais (et les méta-analyses réalisées à partir de ces derniers) par rapport à la population à qui s'adresse la psychothérapie. On peut également songer à la rigidité de la méthode thérapeutique le plus souvent « manualisée » et délivrée selon un protocole bien précis ; rigidité qui somme toute correspond plutôt rarement aux pratiques quotidiennes des psychothérapeutes formés. Sur la base d'une revue de 63 méta-analyses examinant les effets des interventions psychothérapeutiques, Matt et Navarro (1997) relevaient d'ailleurs qu'une des faiblesses importante de la littérature sur le sujet concerne le fait que les thérapeutes et les clients évalués dans ces études ne sont que peu représentatifs de ce que l'on peut observer dans les pratiques cliniques quotidiennes. Sur la base des métaanalyses, la question de l'évaluation de l'efficacité réelle des pratiques psychothérapeutiques dans des contextes moins contrôlées où la clinique dicte la recherche et non l'inverse reste donc ouverte. Récemment, Stiles, Barkham et leurs collègues ont toutefois mis en évidence dans des études naturalistes l'efficience des approches centrées sur la personne, cognitivocomportementales et psychodynamique proposées dans la pratique de routine par le Service 
National de Santé (NHS - National Health Service) en Grande-Bretagne (Barkham, Stiles, Connell, et Mellor-Clark, 2012 ; Stiles, Barkham, Mellor-Clark, et Connell, 2008 ; Stiles, Barkham, Twigg, Mellor-Clark, et Cooper, 2006).

Deuxièmement, la qualité d'une méta-analyse dépend spécifiquement de la qualité des essais contrôlés randomisés inclus dans cette dernière (biais connu sous l'acronyme anglais GIGO pour garbage in garbage out). A titre d'exemple, dans une méta-analyse examinant l'efficacité des thérapies cognitivo-comportementales sur les symptômes positifs (délires et hallucinations) chez des patients psychotiques, Zimmermann, Favrod, Trieu et Pomini (2005) ont pu mettre en évidence que la taille de l'effet obtenue à partir des essais sans évaluation à l'aveugle (i.e. les évaluateurs ne sont pas indépendants et connaissent le traitement dont a bénéficié le patient) est approximativement deux fois plus élevée que celle obtenue à partir d'essais plus rigoureux respectant cette exigence méthodologique. Dans le même registre, un autre problème bien connu de la recherche sur l'efficacité des psychothérapies est le biais d'allégeance, c'est-à-dire le fait que les essais cliniques rapportent généralement un effet supérieur de la psychothérapie évaluée lorsque les auteurs de l'étude sont de l'obédience de l'intervention psychothérapeutique examinée (Despland, Zimmermann, et de Roten, 2006 ; Luborsky, et al., 1999). En effet, contrairement au modèle des études pharmacologiques, où le patient reçoit aléatoirement soit une substance active (condition expérimentale) soit un placebo (condition contrôle) sans que ni lui ni le prescripteur aient connaissance de cette condition, la recherche en double-aveugle n'est pratiquement pas possible en psychothérapie. Il est par ailleurs difficile de s'assurer que les thérapeutes impliqués dans le placebo de psychothérapie (condition contrôle) aient les mêmes attentes par rapport à la réussite de l'intervention que leurs homologues impliqués dans des interventions psychothérapeutiques spécifiques (O’Leary et Borkovec, 1978). Ainsi, les attentes et les croyances des clients et des 
thérapeutes, les processus relationnels ou encore le contexte et le rituel des séances sont des ingrédients actifs de toute relation d'aide dont il s'agirait de tenir compte (INSERM, 2004).

Troisièmement, la méta-analyse, comme n'importe quelle procédure scientifique, peut être alimentée par l'allégeance de l'un ou l'autre de ses rédacteurs. Du reste, dans le débat passionné au sujet de la question de la psychothérapie la plus efficace (question de l'efficacité relative), de nombreuses méta-analyses, bien que basées sur le même paradigme méthodologique et parfois sur les mêmes études, aboutissent à des conclusions radicalement opposées. On peut citer à ce sujet, entre autres, les débats par articles interposés au sujet de la supériorité des thérapies cognitives dans le traitement de la dépression entre Gloagen et Wampold (Gloagen, Cottraux, Cucherat, et Blackburn, 1998 ; Wampold, Minami, Baskin, et Tierney, 2002), ou plus récemment entre Leichsenring et Bhar au sujet de l'efficacité des psychothérapies psychodynamiques de longue durée (Leichsenring et Rabung, 2008 ; Bhar, Thombs, Pignotti, Bassel, Jewett, Coyne, et Beck, 2010). On peut donc assister à une véritable « guerre » des méta-analyses où les tenants de différentes approches théoriques se critiquent réciproquement, parfois de façon très virulente, pour mettre en avant la supériorité de leur école thérapeutique. A ce sujet, l'exemple polémique le plus célèbre dans le monde francophone, dont la prestigieuse revue Science (Holden, 2005) s'est même fait l'écho, fait suite à la publication du rapport de l'INSERM en 2004. Ce rapport affirme notamment qu' « il est désormais clair que des différences significatives existent entre les différentes approches. Les effets les plus positifs sont à porter au crédit des approches cognitives et comportementales dans les comparaisons globales, sachant que les troubles anxieux et les troubles de l'humeur représentent la majorité des syndromes psychiatriques inclus dans ces méta-analyses » (INSERM, 2004, p. 418), alors qu'on trouve une formulation très différente dans la dernière édition du Garfield \& Bergin Handbook of Psychotherapy Research (Lambert et Ogles, 2004, p. 166) considérée comme faisant autorité par la très grande majorité 
des chercheurs en psychothérapie au sein de la communauté internationale : « lorsqu'on examine les études de bonne qualité méthodologique qui comparent les différentes orientations thérapeutiques, on doit constater que les différences sont régulièrement faibles ou négligeables $»^{3}$. C'est ce que l'on a pris l'habitude d'appeler dans la recherche en psychothérapie le Verdict de l'oiseau Dodo (Everybody has won and all must have prizes, Lewis Carroll, 1865), à savoir que toutes les formes de psychothérapies bona fide étudiées se valent.

Quatrièmement, comme nous l'avons déjà laissé entendre, le fait de considérer l'essai contrôlé randomisé comme le dispositif méthodologique privilégié pour étudier l'efficacité des psychothérapies n'est pas exempt de problèmes. En effet, ce type de dispositif méthodologique repose sur des présupposés épistémologiques selon lesquels il est possible d'évaluer objectivement des interventions psychothérapeutiques, conçues comme des techniques, pour des troubles psychiques spécifiques (Freire, 2006). Il semble pourtant évident que des thérapeutes formés à la même approche, même s'ils acceptent de proposer une intervention manualisée, diffèrent les uns des autres dans leur manière d'exercer la psychothérapie (différences inter-individuelles), sans compter que leur pratique varie sans doute d'un client à l'autre et d'une séance à l'autre (différences intra-individuelles) (Kramer, de Roten, et Despland, 2005 ; Najavits et Weiss, 1994 ; Rifkin, 2007). Il est donc discutable de considérer a priori que la psychothérapie puisse être examinée de la même manière qu'une substance active dans une étude pharmacologique.

Cinquièmement, il est généralement admis qu'il existe dans la littérature un biais de publication en vertu duquel les essais cliniques qui mettent en évidence la supériorité d'un traitement sur un autre ont plus de probabilité d'être publiés, que ceux n'indiquant pas de différences entre les traitements (e.g. Begg, 1994 ; Easterbrook, Berlin, Gopalan, et Matthews, 1991). Ce biais de publication résulte aussi bien des chercheurs eux-mêmes qui

\footnotetext{
${ }^{3}$ Traduction de l'auteur
} 
s'autocensurent et gardent les résultats nuls « au fond de leurs tiroirs » (File drawer problem, Rosenthal, 1979), que des éditeurs de revues scientifiques peu enclins à publier des résultats non statistiquement significatifs (Cooper, 2010 ; Greenwald, 1975). Il est évident que ce biais de publication peut entacher la validité d'une méta-analyse (Rosenthal, 1979), puisque le fait de ne pas tenir compte des essais cliniques non publiés peut fausser l'appréciation globale d'un traitement (pour une revue en français des techniques statistiques permettant d'identifier et de corriger ce biais, voir Laroche, 2007).

Last but not least, les résultats des essais contrôlés randomisés et des méta-analyses ne donnent que des indications sur des tendances moyennes sur un ensemble d'individus et ne peuvent ainsi pas être généralisés à un client en particulier (Elliott, 2001 ; Petot, 2002). Autrement dit, même si une intervention psychothérapeutique est considérée efficace sur la base de « résultats probants », il est possible qu'elle soit dénuée d'efficacité avec certains clients. Par conséquent, à vouloir favoriser uniquement les traitements appuyés empiriquement sur la base de méta-analyses ou d'essais cliniques contrôlées, il existe un risque de s'enfermer dans une position dogmatique au mépris de l'expérience du thérapeute, de la singularité de la relation thérapeutique, et des croyances et préférences des clients (voir Tyrell et Poussin, 2005).

Face à ces nombreuses critiques, l'Association Américaine de Psychologie (APA, 2006) a revu les lignes directrices guidant l'évaluation empirique des traitements psychologiques. Ces nouvelles directives reconnaissent l'intérêt de l'utilisation de méthodologies plurielles et diverses, et proposent la définition suivante des pratiques basées sur l'évidence : « c'est l'intégration à l'expertise clinique de la meilleure recherche disponible, tout en tenant compte des caractéristiques, de la culture et des préférences du patient ${ }^{4} »($ APA, 2006, p. 273).

\footnotetext{
${ }^{4}$ Traduction de l'auteur
} 


\section{En guise de conclusion}

Dans la question de l'évaluation de l'effet des psychothérapies, la méta-analyse s'inscrit clairement dans le mouvement des approches fondées sur les données probantes et constitue un dispositif empirique privilégié pour examiner de façon rigoureuse l'efficacité des traitements psychologiques (Chambless et Hollon, 1998). Dépendant d'une « vision médicale » de la psychothérapie, cette initiative a suscité et suscite encore de vives controverses et de nombreuses résistances (Freire, 2006 ; Wampold, 2001).

Il est vrai qu'en raison de sa complexité, l'évaluation quantitative des psychothérapies est considérée par certains psychothérapeutes comme une démarche contraignante, voire impossible. Les échanges avec les cliniciens font parfois apparaître une crainte plus fondamentale, à savoir que l'efficacité des psychothérapies ne pourrait être mise en évidence avec ce type de méthodologies « expérimentales ». Pourtant, il n’en est rien, et aussi réductrice et simplificatrices que soient les démarches des pratiques psychologiques basées sur l'évidence, elles démontrent que les psychothérapies sont des méthodes efficaces et économiques (Zimmermann, et al., 2008).

Même si la pratique clinique s'est trouvée bien peu modifiée - dans le monde francophone tout du moins - par les résultats de ces études empiriques qui ne sont pas exemptes de biais méthodologiques, ces travaux ont le mérite d'inscrire les psychothérapies dans une démarche évaluative rigoureuse avec le souci louable d'améliorer la qualité des interventions proposées (Despland, et al., 2006). Dans un contexte où les systèmes de santé sont de plus en plus marqués par des impératifs économiques, il est par ailleurs probable que les écoles de psychothérapie qui refuseraient toute forme d'évaluation (prioritairement basée sur l'essai clinique randomisé) soient de plus en plus mises à l'écart. Il est pourtant souhaitable que la recherche en psychothérapie fasse preuve d'un pluralisme méthodologique, et n'ait pas comme unique ambition de répondre à la question de l'efficacité (Freire, 2006). Par exemple, 
Miller (2007, cité par MacLeod, Elliott, et Rodgers, 2012) suggère que l'étude de cas est une source d'information cruciale dans la recherche en psychothérapie. L'étude de cas unique connaît du reste un renouveau très important dans la littérature et les revues scientifiques spécialisées (e.g. Elliott, 2001, 2002 ; Fishman, 1999 ; Stiles, 2003 ; Watson, Goldman, et Greenberg, 2007). Parallèlement à ce renouveau, les chercheurs se préoccupent actuellement plus de comprendre ce qui fait que chaque forme de psychothérapie est efficace (ingrédients processuels) en se centrant par exemple sur l'alliance thérapeutique, les caractéristiques du patient, ou encore les qualités personnelles du psychothérapeute indépendamment de son école thérapeutique (Cooper, 2008). Une étude récente de Okiishi, Lambert, Nielsen et Ogles (2003) examinant les effets des thérapeutes sur l'évolution de leurs clients montre par exemple que leurs orientations théoriques (cognitivo-comportementale, humaniste, ou psychodynamique) n’a aucune influence sur les résultats de la psychothérapie.

En conclusion, lorsque l'on aborde les questions d'évaluation des psychothérapies, il est important de garder à l'esprit que « l'analyse de donnée est une aide à la pensée, pas un substitut » (Green et Hall, 1984, p. 52). Par ailleurs, nous pensons que cette évaluation ne peut pas uniquement se réaliser dans des paradigmes épistémologiques importés des sciences naturelles, et qu'elle aurait tout à gagner à défendre la complémentarité des méthodes de recherche et à s'inscrire également dans des paradigmes propres aux sciences humaines. 
Méta-analyse : Faits et fiction

\section{Déclaration d'intérêts}

Les auteurs déclarent ne pas avoir de conflits d'intérêts en relation avec cet article. 


\section{Références}

APA Task Force on Evidence-Based Practice (2006). Evidence-based practice in psychology. American Psychologist, 61, 271-285.

Barkham, M., Stiles, W. B., Connell, J., \& Mellor-Clark, J. (2012). Psychological treatment outcomes in routine NHS services: What do we mean by treatment effectiveness? Psychology and Psychotherapy: Theory, Research and Practice, 85(1), 1-16.

Begg, C. B. (1994). Publication bias. In H. Coopper, \& L. V. Hedges (Eds.), The Handbook of Research Synthesis (p. 399-409). New York: Russel Sage Foundation.

Bergin, A. E. (1971). The evaluation of therapeutic outcomes. In A. E. Bergin \& S. L. Garfield (Eds.), Handbook of Psychotherapy and Behavior Change (pp. 217-270). New York: Wiley.

Bhar, S. S., Thombs, B. D., Pignotti, M., Bassel, M., Jewett, L., Coyne, J. C., \& Beck, A. T. (2010). Is longer-term psychodynamic psychotherapy more effective than shorter-term therapies? Review and critique of the evidence. Psychotherapy and Psychosomatics, 79(4), 208-216.

Borenstein, M., Hedges, L. V., Higgins, J. P. T., \& Rothstein, H. R. (2009). Introduction to meta-analysis. New York : Wiley.

Bradley, R., Greene, J., Russ, E., Dutra, L., \& Westen, D. (2005). A multidimensional metaanalysis of psychotherapy for PTSD. The American Journal of Psychiatry, 162(2), 214-227.

Carr, A. (2009). What works with children, adolescents, and adults? A review of research on the effectiveness of psychotherapy. Hove : Routledge.

Carroll, L. (1865/1971). Alice in wonderland. New York : Norton \& Company.

Chambless, D. L., \& Hollon, S. D. (1998). Defining empirically supported therapies. Journal of Consulting and Clinical Psychology, 66, 7-18.

Cohen, J. (1988). Statistical power analysis for the behavioral sciences (2nd ed.). New York: Academic Press.

Cooper H. M. (2010). Research synthesis and meta-analysis (4th ed.). Los Angeles, CA : Sage. 
Cooper, M. (2008). Essential research findings in counselling and psychotherapy. The facts are friendly. London: Sage.

Cucherat, M. (1997). Méta-analyse des essais thérapeutiques. Paris: Masson.

Despland, J.-N., Zimmermann, G., \& de Roten, Y. (2006). L'évaluation empirique des psychothérapies. Psychothérapies, 26(2), 91-95.

Easterbrook, P. J., Berlin, J. A., Gopalan, R., \& Matthews, D. R. (1991). Publication bias in clinical research. Lancet, 337(8746), 867-872.

Elliott, R. (2001). Hermeneutic single case efficacy design (HSCED): An overview. In K. J. Schneider, J. F. T. Bugental, \& J. F. Fraser (Eds.), Handbook of Humanistic Psychology (pp. 315-324). Thousand Oaks, CA: Sage.

Elliott, R. (2002). Hermeneutic single-case efficacy design. Psychotherapy Research, 12, 121.

Eysenck, H. J. (1952). The effect of psychotherapy: an evaluation. Journal of Consulting Psychology, 16, 319-324.

Eysenck, H. J. (1961). The effect of psychotherapy. In H. J. Eysenck (Ed.), Handbook of Abnormal Psychology (pp. 697-725). New York: Basic Books.

Eysenck, H. J. (1965). The effect of psychotherapy. International Journal of Psychiatry, 1, 97-178.

Eysenck, H. J. (1978). An exercise in mega-silliness. American Psychologist, 33(5), 517.

Eysenck, H. J. (1983). The effectiveness of psychotherapy: the specter at the feast. The Behavioral and Brain Sciences, 6, 290.

Fishman, D. B. (1999). The case for pragmatic psychology. New York: New York University Press.

Freire, E. S. (2006). Randomized controlled clinical trial in psychotherapy research: An epistemological controversy. Journal of Humanistic Psychology, 46(3), 323-335.

Glass, G. V. (1976). Primary, secondary, and meta-analysis. Educational Researcher, 5, 3-8.

Glass, G. V. (2000, January). Meta-analysis at 25. Unpublished manuscript. Retrieved April 25, 2012 from http://www.gvglass.info/papers/meta25.html 
Gloagen, V., Cottraux, J., Cucherat, M., \& Blackburn, I. (1998). A meta-analysis of the effects of cognitive therapy in depressed patients. Journal of Affective Disorders, 49, 5972.

Green, B. F., \& Hall, J. A. (1984). Quantitative methods for literature review. Annual Review of Psychology, 35, 37-53.

Greenwald, A. G. (1975). Consequences of prejudice against the null hypothesis. Psychological Bulletin, 82, 1-20.

Grissom, R. J. (1996). The magical number $.7 \pm .2$ : Meta-meta-analysis of the probability of superior outcome in comparisons involving therapy, placebo, and control. Journal of Consulting and Clinical Psychology, 64(5), 973-982.

Holden, C. (Ed.) (2005). French psychoflap [Random samples]. Science, 307, 1197.

Hunter, J. E, \& Schmidt, F. L. (1990). Methods of meta-analysis: Correcting error and bias in research findings. Newbury Park, CA : Sage.

INSERM. (2004). Psychothérapie. Trois approches évaluées. Expertise Collective INSERM

(O. Canceil, J. Cottraux, B Falissard, M. Flament, J. Miermont, J. Swendsen, M.

Teherani, J.M Thurin). Paris : INSERM.

Kramer, U., de Roten, Y., \& Despland, J.-N. (2005). Les thérapeutes font-ils ce qu'ils disent faire ? Comparaison entre prototypes idéaux et pratiques réelles pour plusieurs formes de psychothérapies. Pratiques Psychologiques, 11(4), 359-370.

Lambert, M. J., Ogles, B. M. (2004). The efficacy and effectiveness of psychotherapy. In M. J. Lambert (Ed.), Bergin and Garfield's Handbook of Psychotherapy and Behavior Change ( $5^{\text {th }}$ ed.) (pp. 139-193). Chichester: John Wiley \& Sons.

Laroche, P. (2007). L'exploration statistique du biais de publication. Journal de la Société Française de Statistique, 148(4), 29-56.

Leichsenring, F., \& Rabung, S. (2008). Effectiveness of long-term psychodynamic psychotherapy: A meta-analysis. JAMA, 300(13), 1551-1565.

Lipsey, M. W., \& Wilson, D. B. (2001). Practical meta-analysis. Thousand Oaks, CA : Sage.

Luborsky, L. (1954). A note on Eysenck's article "The effect of psychotherapy: An evaluation". British Journal of Psychology, 45, 129-131. 
Luborsky, L., Diguer, L., Seligman, D. A., Rosenthal, R., Krause, E. D., Johnson, S., Halperin, G., et al. (1999). The Researcher's own therapy allegiances: A « wild card » in comparisons of treatment efficacy. Clinical Psychology: Science and Practice, 6(1), 95106.

Luborsky, L., Singer, B., \& Luborsky, L. (1975). Comparative studies of psychotherapies. Is it true that « everyone has won and all must have prizes ». Archives of General Psychiatry, 32, 995-1008.

MacLeod, R., Elliott, R., \& Rodgers, B. (2012). Process-experiential/emotion-focused therapy for social anxiety: A hermeneutic single-case efficacy design study. Psychotherapy Research, 22(1), 67-81.

Matt, G. E., \& Navarro, A. M. (1997). What meta-analyses have and have not taught us about psychotherapy effects: A review and future directions. Clinical Psychology Review, 17(1), $1-32$.

Miller, R. B. (2007). In praise of case studies and anecdotal data. Paper presented at SPR Meeting, Madison, Wisconsin.

Najavits, L. M., \& Weiss, R. D. (1994). Variations in therapist effectiveness in the treatment of patients with substance use disorders: an empirical review. Addiction, 89(6), 679-688.

Nathan, P. E., Stuart, S. P., \& Dolan, s. L. (2000). Research on psychotherapy efficacy and effectiveness: Between Scylla and Charybdis. Psychological Bulletin, 126(6), 964-981.

Okiishi, J., Lambert, M. J., Nielsen, S. L., Ogles B. M. (2003). Waiting for supershrink: An empirical analysis of therapists effects. Clinical Psychology \& Psychotherapy, 10, 361-73.

O'Leary, D. K., \& Borkovec, T. D. (1978). Conceptual, methodological, and ethical problems of placebo group in psychotherapy research. American Psychologist, 33(9), 821-830.

Paterson, B.L., Thorne, S.E., Canam, C. \& Jillings, C. (2001). Meta-study of qualitative health research : A practical guide to meta-analysis and metasynthesis. Thousand Oaks : Sage.

Petot, J.-M. (2002). Les différentes méthodes de psychothérapie sont-elles également efficaces quelles que soient les différences entre les patients? Cahiers de Psychologie Clinique, 18(1), 189-205. 
Randolph, J. J. \& Edmondson, R. S. (2005). Using the binomial effect size display (BESD) to present the magnitude of effect sizes to the evaluation audience. Practical Assessment, Research \& Evaluation, 10(14). Available online : http://pareonline.net/getvn.asp?v=10\&n=14

Rifkin, A. (2007). Randomized controlled trials and psychotherapy research. American Journal of Psychiatry, 164(1), 7-8.

Rosenthal R. (1979). The "file drawer problem" and tolerance for null results. Psychological Bulletin, 86, 638-641.

Rosenthal, R. (1990). How are we doing in soft psychology. American Psychologist, 45, 775777.

Rosenthal, R. (1994). Parametric measures of effect size. In H. Cooper \& L. V. Hedges (Eds.), The Handbook of Research Synthesis (pp. 231-244). New York : Russel Sage Foundation.

Rosenthal, R., \& Rubin, D. B. (1982). A simple, general purpose display of magnitude of experimental effect. Journal of Educational Psychology, 74, 166- 169.

Rosenzweig, S. (1954). A transvaluation of psychotherapy : A reply to Hans Eysenck. Journal of Abnormal and Social Psychology, 49, 298-304.

Rosqvist, J., Thomas, J. C., \& Truax, P. (2011). Effectiveness versus efficacy studies. In J. C. Thomas, \& M. Hersen (Eds.), Understanding Research in Clinical and Counseling Psychology (2 $2^{\text {nd }}$ Ed.) (pp. 319-354). New York : Routledge.

Roth, A., \& Fonagy, P. (2005). What works for whom? A critical review of psychotherapy research. New York: Guilford Press.

Sackett, D., Rosenberg, W. M., Gray, J. A., Haynes, R. B., \& Richardson, W. S. (1996). Evidence based medicine: What it is and what it isn't. British Medical Journal, 312, 71-72.

Smith, M. L., \& Glass, G. V. (1977). Meta-analysis of psychotherapy outcome studies. American Psychologist, 32, 752-760.

Steering Committee of the Physicians' Health Study Research Group (1988). Preliminary report: Findings from the aspirin component of the ongoing physicians' health study. New England Journal of Medicine, 318, 262-264. 
Stiles, W. B. (2003). When is a case study scientific research? Psychotherapy Bulletin, 38(1), 6-11.

Stiles, W. B., Barkham, M., Mellor-Clark, J., \& Connell, J. (2008). Effectiveness of cognitive-behavioural, person-centred, and psychodynamic therapies in UK primarycare routine practice: Replication in a larger sample. Psychological Medicine, 38, $677-$ 688.

Stiles, W. B., Barkham, M., Twigg, E., Mellor-Clark, J., \& Cooper, M. (2006). Effectiveness of cognitive-behavioural, person-centred, and psychodynamic therapies as practiced in UK National Health Service settings. Psychological Medicine, 36, 555-566.

Tyrrell J., \& Poussin G. (2005). Quelques réflexions sur l'avenir en France des traitements psychologiques appuyés empiriquement. Psychologie Française, 50(4) 487-500.

Villamaux, M. (2000). La recherche sur l'efficacité des psychothérapies au 20ème siècle ? Une histoire mouvementée. Pour la Recherche, 25, 4. Consultable en ligne : http://psydoc-fr.broca.inserm.fr/recherche/PLR/PLR25/PLR25.html\#6

Wampold, B. E. (2001). The great psychotherapy debate: Models, Methods, and findings. Mahwah, NJ: Lawrence Erlbaum.

Wampold, B. E., Minami, T., Baskin, T. W., \& Tierney, S. C. (2002). A meta - (re) analysis of the effects of cognitive therapy versus "other therapies" for depression. Journal of Affective Disorders, 69, 159-165.

Watson, J. C., Goldman, R. N., \& Greenberg, L. S. (2007). Case studies in emotion-focused treatment of depression: A comparison of good and poor outcome. Washington DC: APA.

Zimmermann, G., de Roten, Y., \& Despland, J.-N. (2008). Efficacité, économicité et caractère approprié de la psychothérapie : Etat de la question. Archives Suisses de Neurologie et de Psychiatrie, 159, 119-126.

Zimmermann, G., Favrod, J. Trieu, V. H., \& Pomini, V. (2005). The effect of cognitive behavioral treatment on the positive symptoms of schizophrenia spectrum disorders : A meta-analysis. Schizophrenia Research, 77(1), 1-9. 
Tableau I. Interprétation des tailles de l'effet (adapté de Wampold, 2001)

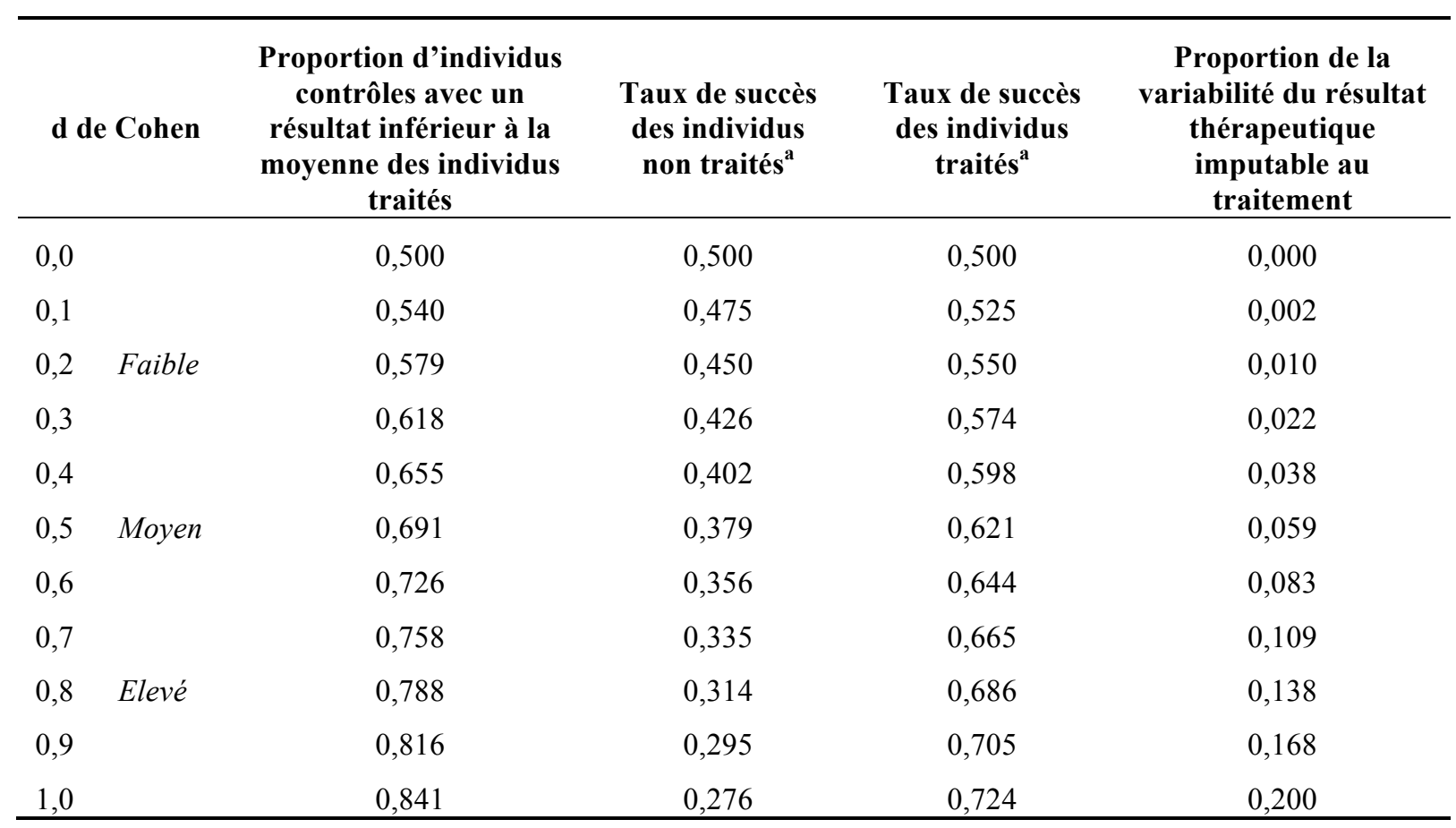

a Binomial effect Size Display (BESD) 